\title{
Development and validation of a prediction rule of major adverse cardiac and cerebrovascular event for high-risk STEMI patients after primary percutaneous coronary intervention
}

\section{Xiaoxiao Zhao}

Diagnosis and Treatment of Acute Coronary Syndrome Group of Yan Hongbing from Fuwai Hospital Chinese Academy of Medical Sciences (CAMS)

Chen Liu

Diagnosis and Treatment of Acute Coronary Syndrome Group of Yan Hongbing from Fuwai Hospital Chinese Academy of Medical Sciences (CAMS)

\section{Peng Zhou}

Diagnosis and Treatment of Acute Coronary Syndrome Group of Yan Hongbing from Fuwai Hospital Chinese Academy of Medical Sciences (CAMS)

\section{Zhaoxue Sheng}

Diagnosis and Treatment of Acute Coronary Syndrome Group of Yan Hongbing from Fuwai Hospital Chinese Academy of Medical Sciences (CAMS)

Jiannan Li

Diagnosis and Treatment of Acute Coronary Syndrome Group of Yan Hongbing from Fuwai Hospital Chinese Academy of Medical Sciences (CAMS)

Jinying Zhou

Diagnosis and Treatment of Acute Coronary Syndrome Group of Yan Hongbing from Fuwai Hospital Chinese Academy of Medical Sciences (CAMS)

\section{Runzhen Chen}

Diagnosis and Treatment of Acute Coronary Syndrome Group of Yan Hongbing from Fuwai Hospital Chinese Academy of Medical Sciences (CAMS)

\section{Ying Wang}

Diagnosis and Treatment of Acute Coronary Syndrome Group of Yan Hongbing from Fuwai Hospital Chinese Academy of Medical Sciences (CAMS)

\section{Yi Chen}

Diagnosis and Treatment of Acute Coronary Syndrome Group of Yan Hongbing from Fuwai Hospital Chinese Academy of Medical Sciences (CAMS)

\section{Li Song}

Diagnosis and Treatment of Acute Coronary Syndrome Group of Yan Hongbing from Fuwai Hospital Chinese Academy of Medical Sciences (CAMS) 


\section{Hanjun Zhao}

Diagnosis and Treatment of Acute Coronary Syndrome Group of Yan Hongbing from Fuwai Hospital Chinese Academy of Medical Sciences (CAMS)

\section{Hongbing Yan ( $\square$ hbyanfuwai2018@163.com )}

Chinese Academy of Medical Sciences \& Peking Union Medical College Fuwai Hospital https://orcid.org/0000-0002-2031-6438

\section{Research}

Keywords: primary percutaneous coronary intervention, high-risk, follow-up, risk prediction score

Posted Date: March 4th, 2021

DOl: https://doi.org/10.21203/rs.3.rs-266770/v1

License: (c) (i) This work is licensed under a Creative Commons Attribution 4.0 International License. Read Full License 


\section{Abstract}

Background and Aims We aimed to develop a clinical prediction tool to improve the prognostication of major adverse cardiac and cerebrovascular events (MACCE) among high-risk myocardial infarction (MI) patients undergoing primary $\mathrm{PCl}$.

Methods Among 4151 consecutive Ml patients who underwent primary percutaneous coronary intervention (PPCI) from FuWai Hospital in BeiJing, China (January 2010 and June 2017), and a prediction rule was derived from derivation and internally validation cohort to predict MACCE after PPCI. Subject must have met at least one clinical criterion and at least one angiographic criterion to be eligible for treatment in the study. The predictive values of markers and clinical variables were assessed with least absolute shrinkage and selection operator (LASSO) regression. The most important variables were included in the score with weights proportional to the model coefficients.

Results The full model included 7 variables, and the risk score was total 160 points. The full model had similar discriminatory value across pre-specified subgroups and was well calibrated. Derivation cohort models predicting MACCE events had $C$ statistics of 0.695 and 0.673 , respectively. The areas under curve (AUC) of the survival receiver operator characteristic curve (ROC) were 0.991 and 0.883 in derivation and validation cohort among 3-year follow-up for predicting the MACCE events. The relative high risk group was observed to have significantly greater likelihood of occurrence of all-caused death, recurrence $\mathrm{MI}$, heart failure, ischemic stroke, hemorrhagic stroke and revascularization compared with the low risk group $(\mathrm{p}<0.05$ respectively).

Conclusion The predicted model was internally validated and calibrated in large cohorts of patients with high risk $\mathrm{Ml}$ receiving primary $\mathrm{PCl}$ therapy to predict the MACCE event and showed modest accuracy in derivation and validation cohorts.

\section{Introduction}

Early primary percutaneous coronary intervention (PPCl) has now been set up as the first-line treatment

for subjects who has acute myocardial infarction (MI) ${ }^{[1]}$. A randomized trial of moderate size ${ }^{[2-7]}$ showed there is a significant increase in major adverse cardio-cerebral events (MACCE) after undergoing the PPCl. Indeed, in high-risk patients and lesion subsets, including those older than 65 years old, with renal dysfunction, diabetes mellitus (DM), thrombotic target lesion and multi-vessel disease邓residual atherothrombotic risk remains substantial. Framingham Heart Study investigators have developed various cardiovascular disease risk prediction project which identified high-risk patients more precise than the conventional classification. It is beneficial and effective that pretreatment risk factors to reduce the risk of cardiovascular disease within patients who are evaluated as high-risk with multivariable prediction equations than treating patients with high levels of single risk factors ${ }^{[8,9]}$. However, few tools are provided to assess the incidence of MACCE events among high-risk MI subjects undergoing primary $\mathrm{PCl}$ to guide long-term risk management. Using these specific data elements, a new risk score project was 
created, with which we sought to: 1) define major independent predictors of MACCE among MI patients with high-risk after undergoing PPCl; and 2) develop and validate a full pre-procedure risk prediction model which adapted to individuals based on precision medicine, healthcare decisions. We present the following article in accordance with the TRIPOD reporting checklist (Appendix file)[1].

[1] The authors have completed the TRIPOD reporting checklist

\section{Material And Methods}

\section{Study population - enrollment and randomization}

This observational, retrospective cohort study analyzed data from Fuwai Hospital, National Center for Cardiovascular Diseases, Peking Union Medical College \& Chinese Academy of Medical Sciences $\varangle$ from a total of 4151 consecutive MI patients who underwent primary PCl at FuWai Hospital in BeiJing, China, between January 2010 and June 2017, were enrolled. 48 patients without follow-up data were excluded from the study. Enrollment into the study will require meeting at least one of the clinical inclusion criteria and one of the angiographic inclusion criteria but none of the exclusion criteria, as shown in figure 1. Clinical criteria included: 1 ) adult patients $\geq 65$ years of age; 2 ) female gender; 3 ) documented PAD or $\mathrm{CAD} / \mathrm{PAD}$ revascularization; 4) diabetes mellitus; 5) chronic kidney disease;6) troponin positive. The angiographic criteria included: 1) multi-vessel coronary artery disease; 2) target lesion requiring total stent length $>30 \mathrm{~mm} ; 3$ ) thrombotic target lesion; 4 ) bifurcation lesions; 5 ) left main ( $\geq 50 \%)$ or proximal LAD $(\geq 70 \%)$ lesion; 6 ) calcified target lesion. These pre-specified criteria were chosen to enroll STEMI patients at high risk for MACCE complications after PCl. Elements are included in validated risks score-projects for either ischemic, bleeding or both types of complications after $\mathrm{PCl}{ }^{[10-18]}$. The R software was used to divide the derivation cohort and the validation cohort randomly and proportionally (70\%:30\%). All patients were referred to the coronary catheterization center with the diagnosis of MI fulfilling the criteria for PPCl according to the guidelines ${ }^{[19,20]}$. The study was approved by the Ethics Committee of Fuwai Hospital, and all patients enrolled will require providing written informed consent for coronary angiography and PPCl. Patient records, including demographics, medical history, physical examination, blood test results, electrocardiography (ECG), echocardiography data, and discharge medication regimen was reviewed. Blood testing was performed at the clinical laboratory in Fuwai Hospital. Experimental protocols and the process for obtaining informed consent were approved by the appropriate by Fuwai hospital institutional review committee. This investigation conformed to the principles outlined in the Declaration of Helsinki. We stated that informed written consent was given prior to the inclusion of subjects in the study.

\section{Definitions and primary outcome}

The primary outcome for this analysis was MACCE which defined as the composite of all-cause death, recurrence myocardial infarction, stroke (including ischemic stroke and hemorrhagic stroke), heart failure or target-vessel revascularization. Hypertension was defined as blood pressure $\geq 140 / 90 \mathrm{mmHg}$ in three occasions at rest or previous diagnosis of hypertension and current use of antihypertensive drugs. 
Diabetes mellitus (DM) was defined according to the 75-g oral glucose tolerance test (OGTT), that is, patients were diagnosed with DM if they met one of the following criteria: (i) fasting plasma glucose level of $\geq 7.0 \mathrm{mmol} / \mathrm{L}$, (ii) 2-h value of $\geq 11.1 \mathrm{mmol} / \mathrm{L}$ in $75-\mathrm{g}$ OGTT, and (iii) casual plasma glucose level of $\geq$ $11.1 \mathrm{mmol} / \mathrm{L}$. Dyslipidemia was defined by any of the following parameters: total cholesterol (TC) 5.0 $\mathrm{mmol} / \mathrm{L}$, low-density lipoprotein cholesterol (LDL-C) $\geq 3.0 \mathrm{mmol} / \mathrm{L}$, triglycerides $(T G) \geq 1.7 \mathrm{mmol} / \mathrm{L}$, highdensity lipoprotein cholesterol (HDL-C) $\geq 1.2 \mathrm{mmol} / \mathrm{L}$ (women) or $\geq 1.0 \mathrm{mmol} / \mathrm{L}$ (men), or statin treatments. Height and weight were measured by trained medical staff; body mass index was calculated by weight $(\mathrm{kg}) /$ height squared $\left(\mathrm{m}^{2}\right)$. No-reflow phenomenon was defined as thrombolysis in myocardial infarction (TIMI) flow grade $<3$ after PPCl. Stroke is defined by the World Health Organization (WHO) Multinational Monitoring of Trends and Determinants in Cardiovascular diseases (MONICA) standard. Stroke is defined as a rapidly developing focal or general brain dysfunction which lasts for more than 24 hours or causes death excluding non-vascular causes (such as trauma, metabolic disorders, tumors and any neurological abnormalities caused by CNS infection). According to the imaging examination in the first week of onset, the neurologist diagnosed stroke included subarachnoid hemorrhage, intracranial hemorrhage, cerebral thrombosis and cerebral embolism. Hemorrhagic stroke includes subarachnoid hemorrhage and intracranial hemorrhage. Furthermore, ischemic stroke includes cerebral thrombosis and cerebral embolism. Transient ischemic attack (TIA) and chronic cerebrovascular disease are not included. The outcome of this study included only initial stroke. Investigators collected data including head CT, head MRI, hospital records from patients during their hospitalization. The STROBE checklist has been provided as a supplementary figure.

\section{Statistical analysis}

The normal distribution of outcome variables was confirmed by Kolmogorov-Smirnov tests. Categorical variables are summarized as frequencies (percentages) and compared with person chi-square tests. Continuous variables are presented as the median and compared using independent t-test. Characteristic of the derivation cohort and validation cohort are showed in table 1 . The study population was randomly split into a development sample consisting of $70 \%$ of admissions and a validation sample consisting of the remaining $30 \%$ of admissions. Baseline patient characteristics and variables from coronary angiography and diagnostic catheterization were considered candidate variables and all prespecified. Candidate variables had $<1.8 \%$ missing data except for the use of IABP (27\%), ApoA (29.2\%) and uric acid (12.6\%). The variables included in the least absolute shrinkage and selection operator (LASSO) regression were showed in the appendix table 1 and it was used to screen the independent variables to draw the corresponding nomogram model.

We developed a predicting model using all potential predictive variables selected by LASSO regression. We also developed a risk prediction score by taking the regression coefficients from the pre-procedure model and assigning them an integer weighted associated with the risk factors. The corresponding nomogram model is drawn according to the regression coefficient of the selected independent variables. For the variables selected in the nomogram model, the values of different variables can correspond to different scores on the integral line at the top of the nomogram (the score range is $0-160$ points) through 
the projection of the vertical line, and the total score can be obtained by adding up the scores corresponding to the values of each variable. The cumulative occurrence probability of MACCE events in 3 and 5 years can be obtained from the total score on the prediction line at the bottom of the nomogram.

In order to reduce the over-fitting bias, the self - sampling method is used to verify the nomogram model. The Harrell's C-statistic was used to compare discrimination between derivation cohort and validation cohort including 3-year and 5-year. Calibration plots were used to access goodness of fit. We draw the survival receiver operator characteristic curve (survival ROC curve) by R language. Survival ROC curves export the best cut-off values and divided into low risk group and high risk group by $\mathrm{R}$ language. We conducted the K-M survival analysis between two groups and export the discrepancy result of the analysis. The subgroup of the K-M curves included the all-caused death, recurrence MI and stroke during 3 and 5 years. LASSO method adopts glmnet package of R language for variable selection, and RMS package of the $\mathrm{R}$ language for drawing and internal verification of nomogram (c-index and calibration chart). The cox regression analysis was performed using the survival package. The main statistical analysis software used in this study is the R language version I 386 3.6.2. Other analyses were performed with SPSS version 20.0 statistical software (SPSS, Inc., Chicago, IL.). A p value $<0.05$ was considered statistically significant. All statistical tests were 2 sided.

\section{Performance and internal validation of new risk prediction equations}

The baseline survival probabilities of each model were obtained by the R language version I 386 3.6.2 commands that were utilized to fit the models. Calibration performance was assessed graphically to predict 3-year and 5-year MACCE events risk and to plot 3-year and 5-year predicted risk against observed 3-year and 5-year risk. A diagonal line with a slope of 1 represents perfect calibration. Observed 3-year and 5-year risk was obtained by the Kaplan-Meier method, and the slopes of regression lines comparing predicted versus observed 5-year risk were calculated. Standard statistical metrics of model and discrimination performance ( $\mathrm{R}^{2}$, Harrell's $\mathrm{C}$ statistic) were calculated. The calibration and discrimination performance of equations developed in the derivation sub-cohort was assessed in the validation subcohort and compared with the performance of models developed in the entire cohort; baseline survival functions and hazard ratios were also compared. Indicators of internal verification include c-index and calibration degree, which respectively represents the prediction accuracy and prediction consistency of the nomogram prediction model. The degree of calibration is represented by a calibration graph. ROC plotting was used for the survival roc package.

\section{Results}

\section{Patient demographics of derivation and validation cohort}

Between Jan 1, 2010, and Jun 30, 2017, the study population included 4151 men and women and excluded 48 people without following up. After applying inclusion criteria, 3404 high-risk MI subjects remained. The average follow up time was 3 year. Subject must have met at least one clinical and at least one angiographic criteria to be eligible for treatment in the study. 2384 people constituted the derivation 
cohort and 1020 cases consisted of validation cohort used in these analyses by random allocation (figure 1). Candidate variables had $<1.8 \%$ missing data except for the use of IABP (27\%), ApoA (29.2\%) and uric acid (12.6\%). Table 1 displays the baseline patient, procedure, and hospital characteristics of the development and validation samples. There were 578 high-risk MI subjects that had MACCE events in derivation cohort after undergoing PPCI procedures, yielding a MACCE event rate of $24.24 \%$. Of these events, $25.09 \%$ were all-caused death; whereas $14.09 \%$ were detected due to recurrence $\mathrm{Ml}, 60.07 \%$ by revascularization, $3.99 \%$ by heart failure, $8.15 \%$ by ischemic stroke and $1.39 \%$ were hemorrhagic stroke.

\section{Screening risk factors for MACCE by LASSO method}

Baseline patient characteristics and variables from coronary angiography and diagnostic catheterization were considered candidate variables and all prespecified (appendix table 1). These variables were filtered by the method of LASSO regression. The filtering and cross-validation processes of independent variables are shown in figure $2 \mathrm{~A} 1$ and $2 \mathrm{~A} 2$ respectively. Lambda. 1 se is the lambda value of the optimal efficiency model in the standard error range which gives a model with excellent performance.

\section{The establishment of risk prediction model}

At this time, a total of 7 independent variables (the subgroup of age, Killip classification, ejection fraction, history of CABG, type of lesions, complete revascularization at admission and multi-vessel disease of coronary artery) were included in the predictive model. The forest plot of the variables which conducted by the multivariate cox regression was shown in the figure $2 \mathrm{~B}$ and the binary decision diagram of the variables was shown in figure $2 \mathrm{C}$. It is necessary to make the classification variables into factorization and then use the as.matrix() function to convert the data from the non-matri $x$ format to the matrix format before the R language "glmet" package can call the data. According to the nomogram model (figure 2D), the score predicting project included 7 variables as the variables of predictive factors.

\section{Clinical Prediction Score}

A simplified risk score was generated to predict MACCE events. The score, ranging from 0 to 160 , assigned points as follows: for patients younger than 40 years, 100 points; for age 40 to younger than 50 years, 80 points; for age 50 to younger than 60 years, 60 points; for age 60 to younger than 70 years, 40 points; for age 70 to younger than 80 years, 20 points; for patients 80 years or older,0; for Killip II, 7.68; for Killip III, 15.36; for Killip IV, 23.03; for EF at admission $\leqq 50 \%, 4.62$; for previous history of CABG, 20; for in-stent restenosis, 4.81; for stent thrombosis, 9.62; for without complete revascularization, 3.45; for multi-vessel lesion, 18 (Figure2E). The elements of clinical prediction score and distribution of score among high-risk $\mathrm{MI}$ patients who undergent $\mathrm{PPCl}$ was shown in figure 2E.

\section{The performance of risk score project}

The MACCE predicting risk model had good discrimination in both the development and validation samples (c-index, development sample 0.695; validation sample 0.673). The model calibration plot for the full model is shown in Figure 3A-D. There was high concordance between the risk predicted by the 
models and the observed MACCE events. Calibration is indicated by the estimated risk against survival from Kaplan- Meier analysis. Gray line represents perfect calibration. Figure 3E-H shows survival (timedependent) ROC curves for the discriminatory value of the 3-year and 5-year evaluation performance of the risk prediction model. The cutoff points of 3-year and 5-year survival ROC curves were 0.22663 , 0.09733 and the area under curve (AUC) were 0.991 and 0.931 in the derivation cohort. On the other hand, the cutoff points of 3-year and 5-year survival ROC curves were $-0.35597,-0.35597$ and AUC were 0.883 and 0.883 in the validation cohort in 3-year and 5-year survival ROC curves. Appendix Figure 1A-D showed the decision curve analysis of 3-year and 5-year in the derivation and validation cohort.

Survival ROC curves export the best cut-off values and divided into relative low risk group and high risk group by R language. We conducted the K-M survival analysis (Figure 4A-P) and export the discrepancy result of the analysis. In the group of predicting MACCE events, the two groups displayed significant difference in both derivation cohort $(p<0.001)$ and validation cohort $(p<0.001)$ shown in figure 4A-D. In the subgroup of predicting all caused death, it is remarkable difference $(p<0.001)$ between the high risk group and relative low risk group in both development and validation group (figure $4 \mathrm{E}-\mathrm{H}$ ). Furthermore, when the endpoint was recurrence $\mathrm{Ml}$, the logrank $\mathrm{p}$ value was less than 0.02 in the 3-year derivation cohort and $\mathrm{p}$ less than 0.01 in the 5-year K-M curve in derivation cohort (figure 4I-L). Finally, we also found distinct discrepancy in predicting the stroke $(p<0.05)$ (figure4 M-P) events in 3-year and 5-year development and validation cohort.

\section{Discussion}

This study developed a clinical prediction score based on clinical and coronary angiology index to help predicting the incidence of long-term MACCE events among MI patients with greater risk factors underwent primary $\mathrm{PCI}$. The MACCE predicting risk model had good discrimination in both the development and validation samples (c-index, validation sample 0.673 ; development sample 0.695 ). For patients who divided into relative low risk group and high risk group by best cut-off values in the prediction model study (derivation cohort), the relative high risk group was observed to have significantly greater likelihood of occurrence of all-caused death, recurrence MI, heart failure, ischemic stroke, hemorrhagic stroke and revascularization compared with the low risk group. These results suggest that it may be possible to identify individual patients with discordant the incidence of MACCE. Although prediction score project is expected to be applied to the subjects represented by enrollment criteria, inconsistent in setting up treatment risks and benefits, adjusting treatment according to personal data, provides opportunities for further optimization results in order to maximize benefits and reduce harm. Yet few equivalent scores are available for use in high-risk patients with acute myocardial infarction undergoing primary $\mathrm{PCI}$ to predict the MACCE events. For these patients, cardiac imaging, coronary angiography and advanced biomarkers are routinely available at admission period, so it is convenient to include them in a score for this setting for long- term management.

The thing that matters, a lot of patient characteristics were correlated with long-term of the incidence of MACCE. Many of the predictive elements which we have identified have been shown in many other 
studies to be predictive of MACCE events. For instance, age is consistently associated with an increased risk of the incidence of MACCE ${ }^{[21]}$, as are other variable like killip classification, EF at admission, history of $C A B G$ and multi-vessels lesion of coronary artery ${ }^{[22]}$. In addition to these factors, we also identified unique variables not present in other predicting models, such as the complete revascularization and the type of lesion detected by the coronary angiography. The addition of such forecasting factors is a noteworthy superiority where the acuity of clinical presentation is generally not as severe compared with previous models that merely included clinical characteristics. It is conspicuous to minimize the risk of the inappropriate care and management for Ml patients with high-risk characteristics who undergoing primary $\mathrm{PCl}$ by including these factors. Bleeding complications including hemorrhagic stroke after primary $\mathrm{PCl}$ are not rare and it is correlated with an increased short-term and long-term risk of mortality $[23,24]$. It has been proposed to drop the hemorrhage among higher-risk patient subjects by the using of vascular closure devices, bivalirudin and radial approach which called bleeding avoidance strategies [25${ }^{28]}$. Therefore, this model can be used to predict the long-term incidence of MACCE events for the high-risk $\mathrm{MI}$ subjects' post-PCl, identify leaders and laggards, and ultimately improve the long-term prognostic of primary $\mathrm{PCl}$ by making healthcare decisions in the follow-up and encouraging the adoption of taking corresponding measures at admission.

According to the present model, the score predicting project included 7 variables (age, Killip classification, ejection fraction, history of CABG, type of lesions, complete revascularization at admission and multivessel disease of coronary artery) as the variables of predictive factors. Previous analyses ${ }^{[29,30]}$ have also identified that the risk factors including age, atrial fibrillation, female sex, killip classification, as well as chronic disease could predict the incidence of stroke within 12-months of PCl which are generally accordance with classic risk variables in the general population. Stroke including ischemic and hemorrhagic are devastating complications with high MACE rates and mortality following PCl. Similarly database derived from the British Cardiovascular Intervention Society (BCIS) has reported ischemic stroke was independently associated with both 30-day mortality and in-hospital MACE by following adjustment for baseline clinical and procedural demographics ${ }^{[31]}$. Previously, Luke $P$ et al ${ }^{[32]}$ demonstrated that the incidence of stroke among outpatients following percutaneous coronary intervention are higher for younger instead of older comparing to the general population. It is coincident with our score projection that age less than 40 years old contributed the greater weight compared with other groups of age. Furthermore, during the beginning period of cardiac catheterization (1970-1980s), the incidence of cerebrovascular events was ranged from $0.03 \%$ to $0.06 \%{ }^{[33]}$ comparing to $0.18 \%-0.44 \%$ during the following years. The increasing in the incidence of stroke-complicating PCI might account for extended use of $\mathrm{PCl}$ and coronary angiography especially among subjects with severe vascular calcification [34]. The time of risk assessment post-event and cardiovascular disease is both the main factors to evaluate the performance of the risk score for secondary prevention. The research of CALIBER [35] enrolled 102023 stable CAD subjects and developed a risk score for people with stable CAD to identify patients at high risk and stand by a management decision. 


\section{Study Limitation}

Several limitations of our study should be considered in interpreting these results. On the basis of the clinical and angiography inclusion and exclusion criteria of the trial, as well as the single-center and retrospective study design, score project of model should be interpreted with the understanding that patients enrolled in clinical trials may not be completely representative of those cared for in routine practice of primary $\mathrm{PCl}$. The analysis ought to be regarded as exploratory despite the predetermination of the score variables. Therefore, the predictive score should be used with circumspection until further external validation is carried on. Optimal and suitable long-term management of procedural and care should be administered independent of the patient's score to reduce overall MACCE events. Furthermore, the extent and severity of granular measures of atherosclerosis were not available and the situation of receiving ticagrelor or other antiplatelet combinations may in part make a difference to the discrimination of the cohort and have a different risk benefit relationship ${ }^{[36]}$. Finally, cerebrovascular events are determined by contacting the subjects followed by validation through medical records. In spite of it is probably to cover almost all hemorrhagic stroke and ischemic strokes, it may undervalue stroke incidence if the patients were asymptomatic and not admitted to hospital.

\section{Conclusion}

In summary, we developed a risk predicted model for estimating long-term (3-year and 5-year) incidence of MACCE based on clinical parameters and indexes of coronary angiography which suit for high-risk subjects with $\mathrm{Ml}$ who underwent primary $\mathrm{PCl}$. The score project can be implemented alongside further medical investigations to support therapeutic decision making. This project requires further prospective assessment to evaluate potential impacts on subjects' management, as well as external validation in other cohorts.

\section{Abbreviations}


I, myocardial infarction

HDL-C, high-density lipoprotein cholesterol

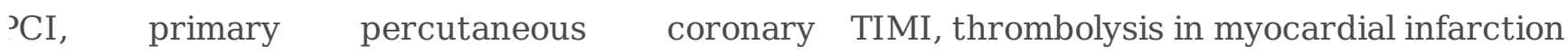

tervention

ŁSSO, least absolute shrinkage and selection WHO, World Health Organization

serator

Э, atrial fibrillation

SBP, systolic blood pressure

JC, areas under curve

TIA, Transient ischemic attack

JC ,survival receiver operator characteristic SE, standard error

trve

JG, electrocardiography

CABG, history of coronary artery bypass grafting

M, Diabetes mellitus

Crea, creatinine

3TT, oral glucose tolerance test

eGFR, estimated glomerular rate

Z, total cholesterol

LPA, lipse activator

J-C, low-density lipoprotein cholesterol

BMI, body mass index

3, triglycerides

PTCA, Percutaneous transluminal coronary angioplasty

BP, diastolic blood pressure

MACCE, major adverse cardiac cerebrovascular events

\section{Declarations}

\section{Ethics approval and consent to participate:}

It is from the ethics committee of the department of Cardiology, Fuwai Hospital, National Center for Cardiovascular Diseases, Peking Union Medical College, China.

\section{Consent for publication}

Written informed consent for publication was obtained from all participants.

\section{Data Availability}

Data have been provided in the supplementary information files that submit alongside the manuscript.

\section{Funding}

This study was supported by the Chinese Academy of Medical Sciences Innovation Fund for Medical Sciences (2016-I2M-1-009), National Natural Science Funds (number: 81970308) and the Fund of "Sanming" Project of Medicine in Shenzhen (number: SZSM201911017). 


\section{Competing interests}

1. We have received funding from Chinese Academy of Medical SciencesInnovation Fund for Medical Sciences (2016-I2M-1-009), National Natural Science Funds (number: 81970308) and the Fund of "Sanming" Project of Medicine in Shenzhen (number: SZSM201911017).

2. Non-financial competing interests.

3. Non-financial competing interests include family associations, political, religious, academic or any other.

\section{Author Contributions}

1. Substantial contributions to conception and design, data acquisition, or data analysis and interpretation: Hongbing Yan, Xiaoxiao Zhao, Chen Liu, Peng Zhou, Zhaoxue Sheng, Jiannan, Jinying Zhou, Runzhen Chen, Ying Wang, Yi Chen, Li Song, Hanjun Zhao,.

2. Drafting the article or critically revising it for important intellectual content: Hongbing Yan, Xiaoxiao Zhao, Chen Liu, Peng Zhou, Zhaoxue Sheng, Jiannan, Jinying Zhou, Runzhen Chen, Ying Wang, Yi Chen, Li Song, Hanjun Zhao.

3. Final approval of the version to be published: Hongbing Yan, Xiaoxiao Zhao, Chen Liu, Peng Zhou, Zhaoxue Sheng, Jiannan, Jinying Zhou, Runzhen Chen, Ying Wang, Yi Chen, Li Song, Hanjun Zhao.

4. Agreement to be accountable for all aspects of the work in ensuring that questions related to the accuracy or integrity of the work are appropriately investigated and resolved: Hongbing Yan, Xiaoxiao Zhao, Chen Liu, Peng Zhou, Zhaoxue Sheng, Jiannan, Jinying Zhou, Runzhen Chen, Ying Wang, Yi Chen, Li Song, Hanjun Zhao.

\section{Acknowledgments}

The authors gratefully acknowledge all individuals who participated in this study.

\section{Disclosures}

No conflicts of interest, financial or otherwise, are declared by the authors.

\section{References}

[1] Borja Ibanez, Stefan James, Stefan Agewall, Manuel J Antunes, Chiara Bucciarelli-Ducci, Héctor Bueno, Alida L P Caforio, Filippo Crea, John A Goudevenos, Sigrun Halvorsen, Gerhard Hindricks, Adnan Kastrati, Mattie J Lenzen, Eva Prescott, Marco Roffi, Marco Valgimigli, Christoph Varenhorst, Pascal Vranckx, Petr Widimský. 2017 ESC Guidelines for the management of acute myocardial infarction in patients presenting with STsegment elevation: the task force for the management of acute myocardial 
infarction in patients presenting with STsegment elevation of the European Society of Cardiology (ESC). Eur Heart J, 2018; 39: 11977.

[2] Stuart J Head, Milan Milojevic, Joost Daemen, Jung-Min Ahn, Eric Boersma, Evald H Christiansen, Michael J Domanski, Michael E Farkouh, Marcus Flather, Valentin Fuster, Mark A Hlatky, Niels R Holm, Whady A Hueb, Masoor Kamalesh, Young-Hak Kim, Timo Mäkikallio, Friedrich W Mohr, Grigorios Papageorgiou, Seung-Jung Park, Alfredo E Rodriguez, Joseph F Sabik 3rd, Rodney H Stables, Gregg W Stone, Patrick W Serruys, A Pieter Kappetei. Stroke Rates Following Surgical Versus Percutaneous Coronary Revascularization [J]. J. Am. Coll. Cardiol, 2018, 72(4):386-398.

[3] Pieter J Vlaar, Tone Svilaas, Iwan C van der Horst, Gilles F H Diercks, Marieke L Fokkema, Bart J G L de Smet, Ad F M van den Heuvel, Rutger L Anthonio, Gillian A Jessurun, Eng-Shiong Tan, Albert J H Suurmeijer, Felix Zijlstra. Cardiac death and reinfarction after 1 year in the thrombus aspiration during percutaneous coronary intervention in acute myocardial infarction Study (TAPAS): a 1-year follow-up study. Lancet 2008; 371:1915-1920.

[4] Ph Gabriel Steg, Stefan K James, Dan Atar, Luigi P Badano, Carina Blömstrom-Lundqvist, Michael A Borger, Carlo Di Mario, Kenneth Dickstein, Gregory Ducrocq, Francisco Fernandez-Aviles, Anthony H Gershlick, Pantaleo Giannuzzi, Sigrun Halvorsen, Kurt Huber, Peter Juni, Adnan Kastrati, Juhani Knuuti, Mattie J Lenzen, Kenneth W Mahaffey, Marco Valgimigli, Arnoud van 't Hof, Petr Widimsky, Doron Zahger. ESC Guidelines for the management of acute myocardial infarction in patients presenting with STsegment elevation. Eur Heart J 2012; 33:2569-2619.

[5] Tim Kinnaird, Thomas Johnson, Richard Anderson, Sean Gallagher, Alex Sirker, Peter Ludman , Mark de Belder, Samuel Copt, Keith Oldroyd, Adrian Banning, Mamas Mamas, Nick Curzen. Intravascular imaging and 12-month mortality after unprotected left main stem PCl: An analysis From the British Cardiovascular Intervention Society Database[J]. JACC. Cardiovascular Interventions, 2020, 10;13(3):346357.

[6] Anna V Kontsevaya, Katie Bates, Henrik Schirmer, Natalia Bobrova David Leon, Martin McKee. Management of patients with acute ST-segment elevation myocardial infarction in Russian hospitals adheres to international guidelines[J]. Open Heart, 2020, 23;7(1):e001134.

[7] Mitsuhiro Takeuchi, Manabu Ogita , Hideki Wada , Daigo Takahashi, Yui Nozaki, Ryota Nishio, Kentaro Yasuda, Norihito Takahashi , Taketo Sonoda, Shoichiro Yatsu, Jun Shitara, Shuta Tsuboi, Tomotaka Dohi, Satoru Suwa, Katsumi Miyauchi, Hiroyuki Daida. Comparison of long-term mortality between living alone patients vs. living together patients with acute coronary syndrome treated with percutaneous coronary intervention[J]. Eur Heart J Qual Care Clin Outcomes. 2020

[8] B Mihaylova, J Emberson, L Blackwell, A Keech, J Simes, E H Barnes, M Voysey, A Gray, R Collins, C Baigent. The effects of lowering LDL cholesterol with statin therapy in people at low risk of vascular disease: meta-analysis of individual data from 27 randomised trials. Lancet 2012; 380: 581-90. 
[9] Sundström J, Jackson R, Neal B, for the BPLTTC. Blood pressure-lowering treatment based on cardiovascular risk: a meta-analysis of individual patient data. Lancet 2014; 384: 591-98.

[10] Si-Hyuck Kang, Keun Ho Park, Hyo-Suk Ahn, Kyung Woo Park, Young Joon Hong, Bon-Kwon Koo, Myung Ho Jeong, Hyo-Soo Kim. Everolimus-eluting versus sirolimus-eluting coronary stents in patients with and without diabetes mellitus. Eurolntervention 2014;10(1):74-82.

[11] Somjot S Brar, John Kim, Simerjeet K Brar, Ray Zadegan, Michael Ree, In-Lu A Liu, Prakash Mansukhani, Vicken Aharonian, Ric Hyett, Albert Yuh-Jer Shen. Long-term outcomes by clopidogrel duration and stent type in a diabetic population with de novo coronary artery lesions. J Am Coll Cardiol 2008; 51(23):2220-7.

[12] Usman Baber, Jeffrey Bander, Rucha Karajgikar, Kapil Yadav, Ali Hadi, Kleanthis Theodoropolous, Nilusha Gukathasan, Swathi Roy, Swapna Sayeneni, Stuart A Scott, Jason C Kovacic, Jennifer Yu, Samantha Sartori, Roxana Mehran, Jaime Uribarri, Juan J Badimon, Paul Muntner, Pedro Moreno, Annapoorna S Kini, Samin K Sharma. Combined and independent impact of diabetes mellitus and chronic kidney disease on residual platelet reactivity. Thromb Haemost 2013;110(1):118-23.

[13] Usman Baber, Roxana Mehran, Ajay J Kirtane, Paul A Gurbel , Georgios Christodoulidis , Akiko Maehara, Bernhard Witzenbichler, Giora Weisz, Michael J Rinaldi, D Christopher Metzger , Timothy D Henry , David A Cox, Peter L Duffy, Ernest L Mazzaferri Jr, Ke Xu, Helen Parise 1 , Bruce R Brodie , Thomas D Stuckey, Gregg W Stone. Prevalence and Impact of High Platelet Reactivity in Chronic Kidney Disease: Results From the Assessment of Dual Antiplatelet Therapy With Drug-Eluting Stents Registry. Circ Cardiovasc Interv 2015;8(6):e001683.

[14] Dominick J Angiolillo, Esther Bernardo, Davide Capodanno, David Vivas, Manel Sabaté, José Luis Ferreiro, Masafumi Ueno, Pilar Jimenez-Quevedo, Fernando Alfonso, Theodore A Bass, Carlos Macaya, Antonio Fernandez-Ortiz. Impact of chronic kidney disease on platelet function profiles in diabetes mellitus patients with coronary artery disease taking dual antiplatelet therapy. J Am Coll Cardiol 2010;55(11):1139-46.

[15] Morrow DA. Cardiovascular risk prediction in patients with stable and unstable coronary heart disease. Circulation 2010;121(24):2681-91.

[16] George D Dangas 1 , Bimmer E Claessen, Roxana Mehran, Ke Xu, Martin Fahy, Helen Parise, José P S Henriques, E Magnus Ohman, Harvey D White, Gregg W Stone. Development and validation of a stent thrombosis risk score in patients with acute coronary syndromes. JACC Cardiovasc Interv 2012;5(11): 1097-105.

[17] Sunil V Rao, Lisa A McCoy, John A Spertus, Ronald J Krone, Mandeep Singh, Susan Fitzgerald, Eric D Peterson. An updated bleeding model to predict the risk of post-procedure bleeding among patients undergoing undergoing percutaneous coronary intervention: a report using an expanded bleeding 
definition from the National Cardiovascular Data Registry CathPCI Registry. JACC Cardiovasc Interv 2013;6(9): 897-904.

[18] Roxana Mehran, Usman Baber, Samin K Sharma, David J Cohen, Dominick J Angiolillo, Carlo Briguori, Jin Y Cha, Timothy Collier, George Dangas , Dariusz Dudek, Vladimír Džavík, Javier Escaned, Robert Gil , Paul Gurbel, Christian W Hamm, Timothy Henry, Kurt Huber, Adnan Kastrati , Upendra Kaul, Ran Kornowski, Mitchell Krucoff, Vijay Kunadian, Steven O Marx, Shamir R Mehta, David Moliterno, E Magnus Ohman, Keith Oldroyd, Gennaro Sardella, Samantha Sartori, Richard Shlofmitz, P Gabriel Steg, Giora Weisz, Bernhard Witzenbichler, Ya-Ling Han, Stuart Pocock, C Michael Gibson. Ticagrelor with or without aspirin in high-risk patients after PCI. N Engl J Med, 2019, 381(21): 2032-2042.

[19] Ph Gabriel Steg, Stefan K James, Dan Atar, Luigi P Badano, Carina Blömstrom-Lundqvist, Michael A Borger, Carlo Di Mario, Kenneth Dickstein, Gregory Ducrocq, Francisco Fernandez-Aviles, Anthony H Gershlick, Pantaleo Giannuzzi, Sigrun Halvorsen, Kurt Huber, Peter Juni, Adnan Kastrati, Juhani Knuuti, Mattie J Lenzen, Kenneth W Mahaffey, Marco Valgimigli, Arnoud van 't Hof, Petr Widimsky, Doron Zahger. ESC guidelines for the management of acute myocardial infarction in patients presenting with STsegment elevation. Eur Heart J 2012; 33:2569-2619.

[20] Patrick T O'Gara, Frederick G Kushner, Deborah D Ascheim, Donald E Casey Jr, Mina K Chung, James A de Lemos, Steven M Ettinger, James C Fang, Francis M Fesmire, Barry A Franklin, Christopher B Granger, Harlan M Krumholz, Jane A Linderbaum, David A Morrow, L Kristin Newby, Joseph P Ornato, Narith Ou, Martha J Radford, Jacqueline E Tamis-Holland, Jacqueline E Tommaso, Cynthia M Tracy, Y Joseph Woo, David X Zhao. 2013 ACCF/AHA guideline for the management of ST-elevation myocardial infarction: executive summary: a report of the American College of Cardiology Foundation/American Heart Association task force on practice guidelines. Circulation 2013; 127: 529-55.

[21] Ce Zhang, Lin Jiang, Lianjun Xu, Jian Tian, Junhao Liu, Xueyan Zhao, Xinxing Feng, Dong Wang, Yin Zhang, Kai Sun, Bo Xu, Wei Zhao, Rutai Hui, Runlin Gao, Jinqing Yuan, Lei Song. Implications of Nterminal pro-B-type natriuretic peptide in patients with three-vessel disease[J]. European Heart Journal (41):40. 3397-3405,

[22] Chengchun Tan, Erfei Luo, Dong Wang, Gaoliang Yan, Yong Qiao, Boqian Zhu, Bo Liu, Jiantong Hou. Usefulness of Haemoglobin Level Combined with CAMI-STEMI Score for Predicting MACCE in Patients with Acute ST-Elevation Myocardial Infarction after PCI[J]. BioMed Research International, 2019, 2019(8):1-8.

[23]Doyle BJ, Rihal CS, Gastineau DA, Holmes DR Jr. Bleeding, blood transfusion, and increased mortality after percutaneous coronary intervention: implications for contemporary practice. J Am Coll Cardiol, 2009;53:2019-27.

[24] Sunil V Rao, Padma R Kaul, Lawrence Liao, Paul W Armstrong, E Magnus Ohman, Christopher B Granger, Robert M Califf, Robert A Harrington, Eric L Eisenstein, Daniel B Mark. Association between 
bleeding, blood transfusion, and costs among patients with non-ST-segment elevation acute coronary syndromes. Am Heart J 2008;155:369-74.

[25]Sherev DA, Shaw RE, Brent BN. Angiographic predictors of femoral access site complications: implication for planned percutaneous coronary intervention. Catheter Cardiovasc Interv 2005;65:196202.

[26] Ajay J Kirtane, Gregory Piazza, Sabina A Murphy, Daniela Budiu, David A Morrow, David J Cohen, Eric Peterson, Nasser Lakkis, Howard C Herrmann, Theresa M Palabrica, C Michael Gibson. Correlates of bleeding events among moderate- to high-risk patients undergoing percutaneous coronary intervention and treated with eptifibatide: observations from the PROTECT-TIMI-30 trial. J Am Coll Cardiol 2006; 47:2374-9.

[27] Freek W A Verheugt, Steven R Steinhubl, Martial Hamon, Harald Darius, Philippe Gabriel Steg, Marco Valgimigli, Steven P Marso, Sunil V Rao, Anthony H Gershlick, A Michael Lincoff, Roxana Mehran, Gregg W Stone. Incidence, prognostic impact, and influence of antithrombotic therapy on access and nonaccess site bleeding in percutaneous coronary intervention. J Am Coll Cardiol Intv 2011;4:191-7.

[28] Sunil V Rao, Fang-Shu Ou, Tracy Y Wang, Matthew T Roe, Ralph Brindis, John S Rumsfeld, Eric D Peterson. Trends in the prevalence and outcomes of radial and femoral approaches to percutaneous coronary intervention: a report from the national cardiovascular data registry. J Am Coll Cardiol Intv 2008;1:379-86.

[29] Emelia J Benjamin, Michael J Blaha, Stephanie E Chiuve, Mary Cushman, Sandeep R Das, Rajat Deo, Sarah D de Ferranti, James Floyd, Myriam Fornage, Cathleen Gillespie, Carmen R Isasi, Monik C Jiménez, Lori Chaffin Jordan, Suzanne E Judd, Daniel Lackland, Judith H Lichtman, Lynda Lisabeth, Simin Liu, Chris T Longenecker, Rachel H Mackey, Kunihiro Matsushita, Dariush Mozaffarian, Michael E Mussolino, Khurram Nasir, Robert W Neumar, Latha Palaniappan, Dilip K Pandey, Ravi R Thiagarajan, Mathew J Reeves, Matthew Ritchey, Carlos J Rodriguez, Gregory A Roth, Wayne D Rosamond, Comilla Sasson, Amytis Towfighi, Connie W Tsao, Melanie B Turner, Salim S Virani, Jenifer H Voeks, Joshua Z Willey, John T Wilkins, Jason Hy Wu, Heather M Alger, Sally S Wong, Paul Muntner. Heart disease and stroke statistics2017 update: a report from the American Heart Association. Circulation 2017; 135: e146-e603.

[30] Martin J O'Donnell, Siu Lim Chin , Sumathy Rangarajan, Denis Xavier, Lisheng Liu, Hongye Zhang, Purnima Rao-Melacini, Xiaohe Zhang, Prem Pais, Steven Agapay, Patricio Lopez-Jaramillo, Albertino Damasceno, Peter Langhorne, Matthew J McQueen , Annika Rosengren, Mahshid Dehghan, Graeme J Hankey, Antonio L Dans , Ahmed Elsayed, Alvaro Avezum, Charles Mondo, Hans-Christoph Diener, Danuta Ryglewicz, Anna Czlonkowska, Nana Pogosova, Christian Weimar, Romaina Iqbal, Rafael Diaz , Khalid Yusoff , Afzalhussein Yusufali , Aytekin Oguz , Xingyu Wang , Ernesto Penaherrera, Fernando Lanas, Okechukwu S Ogah, Adesola Ogunniyi, Helle K Iversen, German Malaga, Zvonko Rumboldt, Shahram Oveisgharan, Fawaz Al Hussain, Daliwonga Magazi , Yongchai Nilanont, John Ferguson, Guillaume 
Pare, Salim Yusuf. Global and regional effects of potentially modifiable risk factors associated with acute stroke in 32 countries (interstroke): a case-control study. Lancet 2016; 388: 761-775.

[31] Chun Shing Kwok, Evangelos Kontopanteli , Phyo K Myint, Azfar Zaman , Colin Berry , Bernard Keavney, Jim Nolan, Peter F Ludman, Mark A de Belder, lain Buchan, Mamas A Mamas. Stroke following percutaneous coronary intervention: type-specific incidence, outcomes and determinants seen by the British Cardiovascular Intervention Society 2007-12[J]. European Heart Journal, 2015, 36, 1618-1628.

[32] Luke P Dawson, Justin A Cole, Terase F Lancefield, Andrew E Ajani, Nick Andrianopoulos, Amanda G Thrift, David J Clark, Angela L Brennan, Melanie Freeman , Jessica O'Brien, Martin Sebastian, William Chan, James A Shaw , Diem Dinh, Christopher M Reid, Stephen J Duffy. Lancefield Incidence and risk factors for stroke following percutaneous coronary intervention. International Journal of Stroke, 2020(0) $1-14$.

[33]Bredlau CE, Roubin GS, Leimgruber PP, Douglas JS, King SB, Gruentzig AR. In-hospital morbidity and mortality in patients undergoing elective coronary angioplasty.Circulation. 1985; 72:1044-1052.

[34] Hoffman SJ, Routledge HC, Lennon RJ, Mustafa MZ, Rihal CS, Gersh BJ, Holmes DR, Gulati R. Procedural factors associated with percutaneous coronary intervention-related ischemic stroke.JACC Cardiovasc Interv. 2012; 5:200-206

[35] Eleni Rapsomaniki, Anoop Shah, Pablo Perel, Spiros Denaxas, Julie George, Owen Nicholas, Ruzan Udumyan, Gene Solomon Feder, Aroon D Hingorani, Adam Timmis, Liam Smeeth, Harry Hemingway. Prognostic models for stable coronary artery disease based on electronic health record cohort of 102023 patients. Eur Heart J 2014; 35:844-52.

[36] Robert W Yeh, Eric A Secemsky, Dean J Kereiakes, Sharon-Lise T Normand, Anthony H Gershlick, David J Cohen, John A Spertus, Philippe Gabriel Steg , Donald E Cutlip, Michael J Rinaldi, Edoardo Camenzin, William Wijns, Patricia K Apruzzese, Yang Song, Joseph M Massaro, Laura Mauri.

Development and validation of a prediction rule for benefit and harm of dual antiplatelet therapy beyond 1 year after percutaneous coronary intervention.[J]. JAMA, 2016, 315(16):1735-1749.

\section{Table}

Table 1 The characteristic of derivation cohort and validation cohort 


\begin{tabular}{|c|c|c|c|c|c|c|}
\hline \multirow[t]{2}{*}{ Variables } & \multicolumn{3}{|c|}{ Derivation cohort $(\mathrm{n}=2384)$} & \multicolumn{3}{|c|}{ Validation cohort $(\mathrm{n}=1020)$} \\
\hline & $\begin{array}{l}\text { MACCE } \\
(n=578)\end{array}$ & $\begin{array}{l}\text { No MACCE } \\
(n=1806)\end{array}$ & Pvalue & $\begin{array}{l}\text { MACCE } \\
(\mathrm{n}=251)\end{array}$ & $\begin{array}{l}\text { No } \\
\text { MACCE } \\
(\mathrm{n}=769) \\
\end{array}$ & Pvalue \\
\hline $\begin{array}{l}\text { Age (years) } \\
\text { Male }[\%(n)]\end{array}$ & \multirow{5}{*}{$\begin{array}{l}65.1246 \\
405 \\
(70.07 \%) \\
167.7623 \\
73.1232 \\
25.8476 \\
79.5655\end{array}$} & \multirow{5}{*}{$\begin{array}{l}58.8632 \\
1412 \\
(78.18 \%) \\
168.4187 \\
73.8718 \\
25.9597 \\
77.3499\end{array}$} & $\begin{array}{l}<0.0001 \\
<0.0001\end{array}$ & \multirow{5}{*}{$\begin{array}{l}64.7331 \\
177 \\
(70.52 \%) \\
168.0256 \\
73.6537 \\
25.9623 \\
80.5840\end{array}$} & \multirow{5}{*}{$\begin{array}{l}58.6736 \\
577 \\
(75.03 \%) \\
168.2891 \\
73.5102 \\
25.8643 \\
76.1917\end{array}$} & $\begin{array}{l}<0.0001 \\
0.1572\end{array}$ \\
\hline Height $(\mathrm{cm})$ & & & 0.0718 & & & 0.6317 \\
\hline Weight (kg) & & & & & & 0.8778 \\
\hline BMI $(\mathrm{kg} / \mathrm{m} \square)$ & & & 0.5528 & & & 0.7119 \\
\hline $\begin{array}{l}\text { Heart rate (beats per } \\
\text { minute) }\end{array}$ & & & 0.0033 & & & 0.0001 \\
\hline SBP (mmHg) & \multirow{8}{*}{$\begin{array}{l}124.6982 \\
73.0745 \\
385 \\
(66.61 \%) \\
234 \\
(40.48 \%) \\
515 \\
(89.10 \%) \\
342 \\
(62.75 \%) \\
98 \\
(16.96 \%) \\
11(1.90 \%) \\
48(8.30 \%)\end{array}$} & \multirow{8}{*}{$\begin{array}{l}124.3561 \\
74.4174 \\
1069 \\
(59.19 \%) \\
677 \\
(37.49 \%) \\
1672 \\
(92.58 \%) \\
1083 \\
(66.08 \%) \\
273 \\
(15.12 \%) \\
19(1.05 \%) \\
85(4.71 \%)\end{array}$} & 0.7031 & \multirow{9}{*}{$\begin{array}{l}121.6345 \\
72.3109 \\
172 \\
(68.53 \%) \\
98 \\
(39.04 \%) \\
226 \\
(90.04 \%) \\
149 \\
(63.14 \%) \\
44 \\
(17.53 \%) \\
8(3.19 \%) \\
28 \\
(11.16 \%) \\
36 \\
(14.34 \%)\end{array}$} & \multirow{8}{*}{$\begin{array}{l}126.1836 \\
74.2051 \\
465 \\
(60.47 \%) \\
277 \\
(36.02 \%) \\
727 \\
(94.54 \%) \\
455 \\
(65.56 \%) \\
131 \\
(17.04 \%) \\
6(0.78 \%) \\
41(5.33 \%)\end{array}$} & 0.1725 \\
\hline $\begin{array}{l}\mathrm{DBP}(\mathrm{mmHg}) \\
\text { Hvnertension }[\%(\mathrm{n})]\end{array}$ & & & & & & 0.0470 \\
\hline Hypertension[\%(n)] & & & 0.0015 & & & 0.0221 \\
\hline Diabetes[\%(n)] & & & 0.1966 & & & 0.3884 \\
\hline Hyperlipidemia[\%(n)] & & & 0.0082 & & & 0.0125 \\
\hline Smoking[\%(n)] & & & 0.1580 & & & 0.4998 \\
\hline Previous PCI[\%(n)] & & & 0.2885 & & & 0.8567 \\
\hline $\begin{array}{l}\text { Previous CABG[\%(n)] } \\
\text { Atrial fibrillation[\%(n)] }\end{array}$ & & & $\begin{array}{l}0.1101 \\
0.0010\end{array}$ & & & $\begin{array}{l}0.0044 \\
0.0014\end{array}$ \\
\hline $\mathrm{CKD}[\%(\mathrm{n})]$ & $\begin{array}{l}64 \\
(11.07 \%)\end{array}$ & $146(8.08 \%)$ & 0.0274 & & $59(7.67 \%)$ & 0.0016 \\
\hline $\begin{array}{l}\text { Laboratory examinations } \\
\text { HDL-cholesterol } \\
(\mathrm{mg} / \mathrm{dl})\end{array}$ & 1.6936 & 1.7091 & 0.7880 & 1.6525 & 1.7000 & 0.5692 \\
\hline LDL-cholesterol (mg/dl) & 2.6939 & & 0.2561 & & 2.7219 & 0.8267 \\
\hline Triglyceride & 1.05 & & & & & 0.3483 \\
\hline LPA (g/L) & 272. & & 0.5234 & & & 0.6753 \\
\hline hs-CRP & & & & & 7.1744 & 0.0001 \\
\hline D-dimer & & & & & & 0.0122 \\
\hline Crea & & 937 & & & & 0.0006 \\
\hline eGFR & & & & & & \\
\hline \multicolumn{7}{|l|}{$\begin{array}{l}\text { Discharge medication } \\
\text { regimen }\end{array}$} \\
\hline Statin[\%(n)] & \multirow{10}{*}{$\begin{array}{l}533 \\
(95.01 \%) \\
554 \\
(98.75 \%) \\
493 \\
(87.88 \%) \\
65 \\
(11.84 \%) \\
304 \\
(54.19 \%) \\
54(9.63 \%) \\
479 \\
(85.38 \%) \\
197 \\
(35.12 \%) \\
142 \\
(25.31 \%) \\
558 \\
(99.47 \%)\end{array}$} & \multirow{10}{*}{$\begin{array}{l}1682 \\
(93.86 \%) \\
1782 \\
(99.44 \%) \\
1353 \\
(75.50 \%) \\
426 \\
(23.81 \%) \\
1134 \\
(63.28 \%) \\
165(9.21 \%) \\
1574 \\
(87.83 \%) \\
531 \\
(29.63 \%) \\
407 \\
(22.71 \%) \\
(778 \\
(99.22 \%)\end{array}$} & 0.3128 & \multirow{10}{*}{$\begin{array}{l}221 \\
(90.57 \%) \\
237 \\
(97.13 \%) \\
205 \\
(84.02 \%) \\
37 \\
(15.48 \%) \\
137 \\
(56.15 \%) \\
18(7.38 \%) \\
211 \\
(86.48 \%) \\
96 \\
(39.34 \%) \\
61 \\
(25.00 \%) \\
242 \\
(99.18 \%)\end{array}$} & \multirow{10}{*}{$\begin{array}{l}711 \\
(92.70 \%) \\
759 \\
(98.96 \%) \\
575 \\
(74.97 \%) \\
184 \\
(24.02 \%) \\
501 \\
(65.32 \%) \\
52(6.78 \%) \\
681 \\
(88.79 \%) \\
193 \\
(25.16 \%) \\
151 \\
(19.69 \%) \\
759 \\
(98.96 \%)\end{array}$} & 0.2814 \\
\hline Aspirin[\%(n)] & & & 0.0923 & & & 0.0399 \\
\hline Clopidogrel & & & $<0.0001$ & & & 0.0034 \\
\hline Ticagrelor [\%(n)] & & & $<0.0001$ & & & 0.0054 \\
\hline ACEI $[\%(n)]$ & & & 0.0001 & & & 0.0097 \\
\hline $\mathrm{ARB}[\%(\mathrm{n})]$ & & & 0.7661 & & & 0.7488 \\
\hline Beta-Blockers[\%(n)] & & & & & & \\
\hline Diuretic[\%(n)] & & & 0.0142 & & & $<0.0001$ \\
\hline Spironolactone[\%(n)] & & & 0.2039 & & & 0.0758 \\
\hline P2Y12 inhibitors & & & 0.5474 & & & 0.7588 \\
\hline $\begin{array}{l}\text { Endpoint events } \\
\text { All caused death [\%(n)] }\end{array}$ & \multirow{3}{*}{$\begin{array}{l}145 \\
(25.09 \%) \\
81 \\
(14.09 \%) \\
346\end{array}$} & $0(0.00 \%)$ & $<0.0001$ & \multirow{3}{*}{$\begin{array}{l}68 \\
(27.09 \%) \\
37 \\
(14.74 \%) \\
144\end{array}$} & $0(0.00 \%)$ & $<0.0001$ \\
\hline Recurrent MI [\%(n)] & & $0(0.00 \%)$ & $<0.0001$ & & $0(0.00 \%)$ & $<0.0001$ \\
\hline revascularization [\% & & $0(0.00 \%)$ & $<0.0001$ & & $0(0.00 \%)$ & $<0.0001$ \\
\hline
\end{tabular}


$(\mathrm{n})]$

heart failure $[\%(\mathrm{n})] \quad 23(3.99 \%)$ ischemic stroke $[\%(\mathrm{n})]$ hemorrhagic stroke $[\%$

$47(8.15 \%)$

$8(1.39 \%)$
$(57.37 \%)$

(n)]

Coronary angiography

\begin{tabular}{|c|c|c|c|c|c|c|}
\hline $\begin{array}{l}\text { Bifurcation lesion }[\% \\
\text { n)] }\end{array}$ & $\begin{array}{l}189 \\
(33.69 \%)\end{array}$ & 630 & 0.5246 & 74 & 272 & 0.1409 \\
\hline $\begin{array}{l}\text { Muli-vessel lesions [\% } \\
\text { n)] }\end{array}$ & $\begin{array}{l}487 \\
(86.81 \%)\end{array}$ & $\begin{array}{l}1339 \\
(74.72 \%)\end{array}$ & $<0.0001$ & $218(89.35)$ & $559(72.99)$ & $<0.0001$ \\
\hline LM lesion [\%(n)] & $\begin{array}{l}58 \\
(10.34 \%)\end{array}$ & $104(5.80 \%)$ & 0.0002 & $23(9.43 \%)$ & $48(6.26 \%)$ & 0.0916 \\
\hline PTCA & $\begin{array}{l}504 \\
(89.84 \%)\end{array}$ & $\begin{array}{l}1566 \\
(87.39 \%)\end{array}$ & 0.1193 & $\begin{array}{l}215 \\
(88.11 \%)\end{array}$ & $\begin{array}{l}681 \\
(88.79 \%)\end{array}$ & 0.7731 \\
\hline Thrombus aspiration & $\begin{array}{l}208 \\
(37.08 \%)\end{array}$ & $\begin{array}{l}784 \\
(43.75 \%)\end{array}$ & 0.0052 & $\begin{array}{l}100 \\
(40.98 \%)\end{array}$ & $\begin{array}{l}323 \\
(42.11 \%)\end{array}$ & 0.7556 \\
\hline $\begin{array}{l}\text { Coronary stent } \\
\text { mplantation }\end{array}$ & $\begin{array}{l}484 \\
(86.27 \%)\end{array}$ & $\begin{array}{l}1593 \\
(88.90 \%)\end{array}$ & 0.0923 & $\begin{array}{l}207 \\
(84.84 \%)\end{array}$ & $\begin{array}{l}680 \\
(88.66 \%)\end{array}$ & 0.1130 \\
\hline The use of IABP & $\begin{array}{l}74 \\
(13.19 \%)\end{array}$ & $168(9.38 \%)$ & 0.0094 & $\begin{array}{l}35 \\
(14.34 \%)\end{array}$ & $68(8.87 \%)$ & 0.0137 \\
\hline
\end{tabular}

Continuous data are presented as mean, categorical variables are presented as \% (n). BMI, body mass index; SBP, systolic blood pressure; DBP, diastolic blood pressure; PCI, percutaneous coronary intervention; CABG, coronary artery bypass grafting; CKD, chronic kidney disease; HDL-C, high-density lipoprotein cholesterol; LDLC, low-density lipoprotein cholesterol; TG, triglyceride; LPA, lipse activator; hs-CRP, high sensitive C-reactive protein; eGFR, estimated glomerular filtration rate; ACEI, angiotensin-converting enzyme inhibitor; ARB, angiotensin receptor blocker; MACCE, major adverse cardiovascular cerebrovascular event

\section{Figures}


Angiographic criteria (Must meet at least one) Multi-vessel coronary artery disease $(n=2976)$

Target lesion requiring total stent length $>30 \mathrm{~mm}$ ( $n=1085$ )

Thrombotic target lesion ( $n=1574$ )

Bifurcation lesions ( $n=1356)$

Left main $(250 \%)$ or proximal LAD $(270 \%)$ lesion $(n=1774)$

Calcified target lesion ( $n=1369)$
Lost to follow-up ( $\mathrm{N}=48$ )

\section{3 cases remained}

Clinical criteria (Must meet at least one)

Adult patients 265 years of age $(n=1350)$

Female gender ( $n=881$ )

Documented PAD or CAD/PAD revascularization ( $n=654$ )

Diabetes mellitus ( $n=1347$ )

Chronic kidney disease $(n=328)$

Troponin positive $(n=1860)$

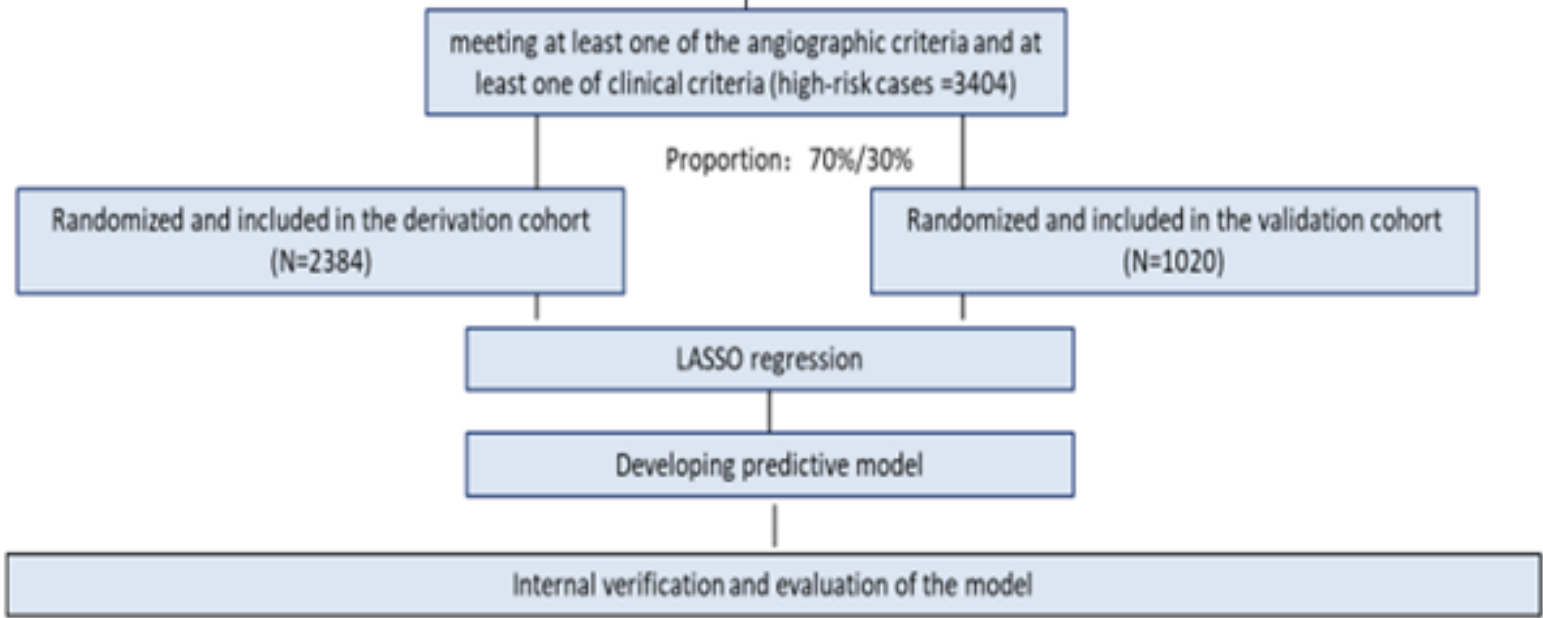

Figure 1 Study Sample Selection Flow Diagram

\section{Figure 1}

Study Sample Selection Flow Diagram 


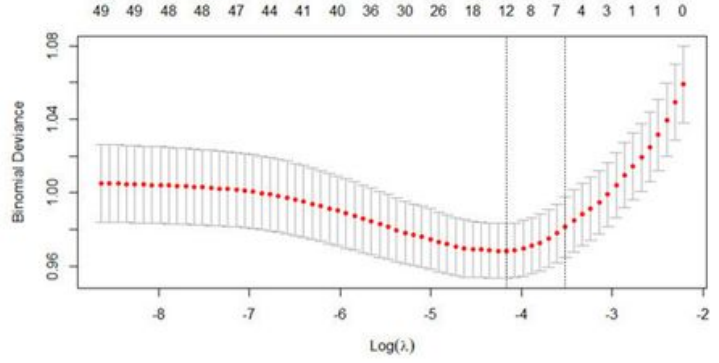

Figure 2 A2 Least absolute shrinkage and selection operator (LASSO) regression

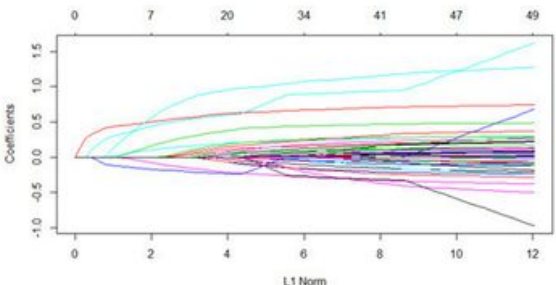

The fitering and cross-validation processes of independent variables are shown in figure $2 \mathrm{~A} 1$ and $2 \mathrm{A2} 2$ respectively. Lambda.1se is the lambda value of the optimal efficiency model in the standard error range which gives a model with excellent performance.

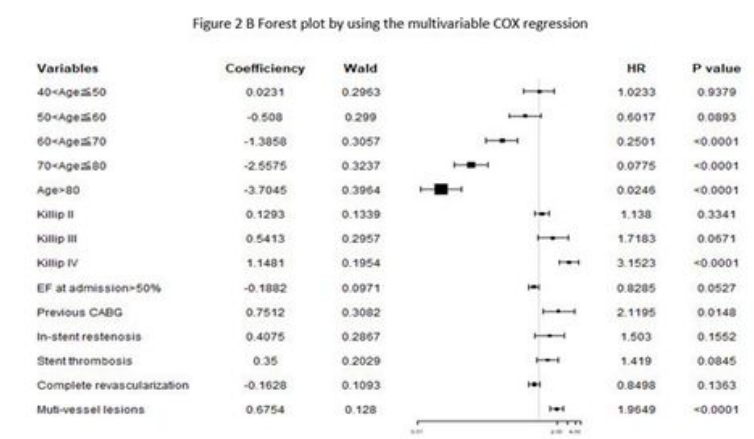

Forest plot by using the multivariable COX regression; $\mathrm{HR}$, hazard ratio; $\mathrm{CABG}$, coronary artery bypass grafting Figure $2 \mathrm{CDecision}$ tree flow diagram

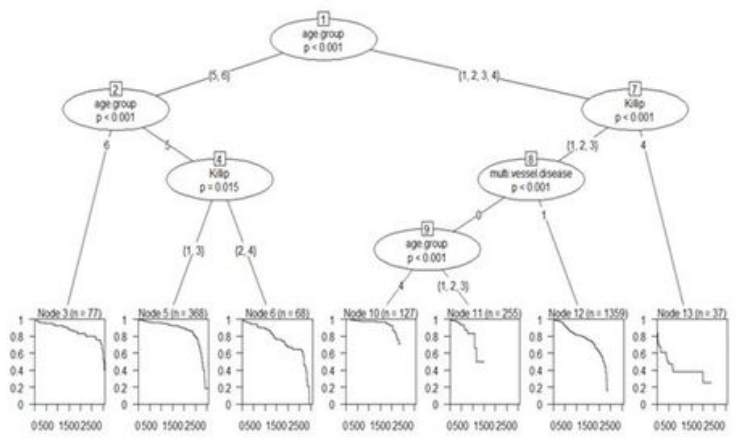

The binary decision diagram of the variables was shown in figure $2 \mathrm{C}$. ports

agegrou

Kap

EF

nistory a CAGB:

Types diesions

Complete revascilarizasion

mat vessel dasease

Tous Poirts

Linew Predctor

30 dary somal

3 year sunval

Spear somal

7 year sormal

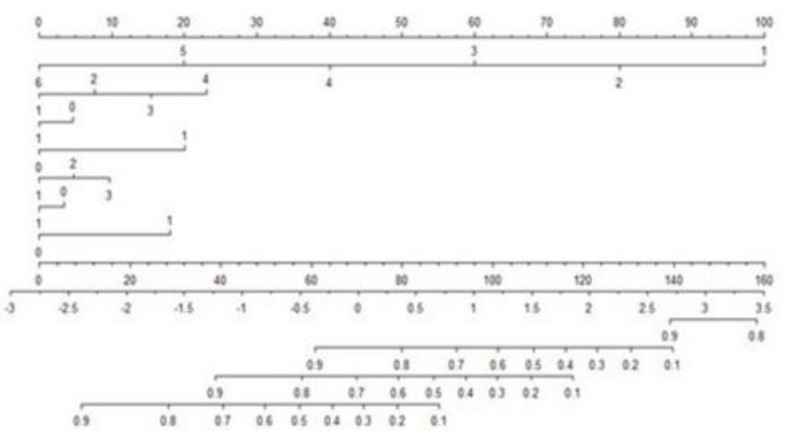

The score, ranging from 0 to 160 , assigned points as follows: for patients younger than 40 years=1, 100 points; for age 40 to younger than 50 years $=2,80$ points; for age 50 to younger than 60 years $=3,60$ points; for age 60 to younger than 70 years $=4,40$ points; for age 70 to younger than 80 years=5, 20 points; for patients 80 years or older=6,0; for Killip II, 7.68; for Killip III, 15.36; for Killip IV, 23.03; for EF at admission $550 \%, 4.62$; for previous history of CABG, 20; for in-stent restenosis, 4.81; for stent thrombosis, 9.62; for without complete revascularization, 3.45; for multi-vessel lesion, 18. Age group, 1 stand for age less than 40 years/ 2 stand for age range from 40 to 50 years/ 3 stand for age range from 50 to 60 years/4 stand for age range from 60 to 70 years $/ 5$ stand for age range from 70 to 80 years/ 6 age stand for age more than 80 years. Killip classification, $1=$ Killip I, 2= Killip II, 3= Killip III, 4=Killip IV. EF, 0 stands for >50\%, 1 stands for less than 50\%. History of CABG, 1=with, 0=without; type of lesion, 1= Denovo lesion, 2=restenosis, 3= stent thrombosis; complete revascularization, $0=$ without, 1=with; mutivessel disease, 1"with, 0-without.

Figure $2 \mathrm{E}$ the internal validation of the model

\begin{tabular}{|c|c|}
\hline $\begin{array}{l}\text { Clinical prediction score } \\
\text { variable }\end{array}$ & Points \\
\hline \multicolumn{2}{|l|}{ Age, y } \\
\hline Age $\leq 40 \ldots 1$ & 100 \\
\hline $40<A g e \leq 50-2$ & 80 \\
\hline $50<A_{g e} \leq 60 \ldots-\ldots$ & 60 \\
\hline $60<$ Age $\leq 70 \ldots-4$ & 40 \\
\hline $70<A g e \leq 80$ & 20 \\
\hline Age $>80$ & 0 \\
\hline Killip II-_-_-_-_-2 & 7.68 \\
\hline Killip III-_-_-_-3 & 15.36 \\
\hline Killip IV & 23.03 \\
\hline EF at admission $\leq 50 \%-0$ & 4.62 \\
\hline Previous CABG 1 & 20 \\
\hline In-stent restenosis $-\cdots-2$ & 4.81 \\
\hline Stent thrombosis- 3 & 9.62 \\
\hline \multicolumn{2}{|l|}{ revascularization -0} \\
\hline $\begin{array}{l}\text { Multi-vessel } \\
\text { lesions---1-1 }\end{array}$ & 18 \\
\hline Total score range: 0 & 160 \\
\hline
\end{tabular}

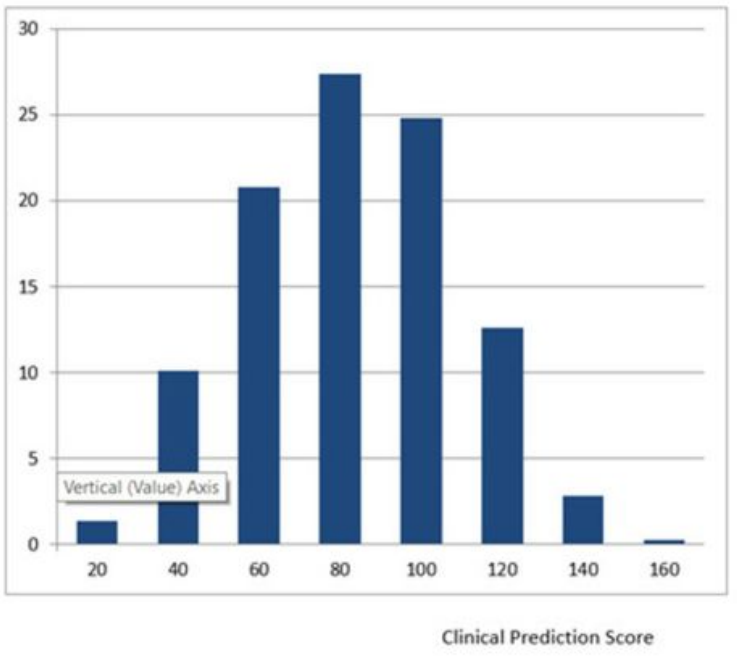

The left side has shown the clinical prediction score variable and corresponding points. The right side has shown the graph of distribution of the clinical prediction score.

\section{Figure 2}

The establishment procedure of the model Figure 2A1, A2 Least absolute shrinkage and selection operator (LASSO) regression. The filtering and cross-validation processes of independent variables are shown in figure $2 \mathrm{~A} 1$ and $2 \mathrm{~A} 2$ respectively. Lambda. 1 se is the lambda value of the optimal efficiency model in the standard error range which gives a model with excellent performance. Figure 2B, Forest plot by using the multivariable COX regression; $\mathrm{HR}$, hazard ratio; $\mathrm{CABG}$, coronary artery bypass grafting Figure $2 \mathrm{C}$, Decision tree flow diagram. The binary decision diagram of the variables was shown in figure $2 \mathrm{C}$. 
Figure 2D, the risk score nomogram. The score, ranging from 0 to 160, assigned points as follows: for patients younger than 40 years, 100 points; for age 40 to younger than 50 years, 80 points; for age 50 to younger than 60 years, 60 points; for age 60 to younger than 70 years, 40 points; for age 70 to younger than 80 years, 20 points; for patients 80 years or older,0; for Killip II, 7.68; for Killip III, 15.36; for Killip IV, 23.03; for $E F$ at admission $\leqq 50 \%, 4.62$; for previous history of CABG, 20; for in-stent restenosis, 4.81 ; for stent thrombosis, 9.62; for without complete revascularization, 3.45; for multi-vessel lesion, 18. Age group, 1 stand for age less than 40 years/ 2 stand for age range from 40 to 50 years/ 3 stand for age range from 50 to 60 years/ 4 stand for age range from 60 to 70 years/ 5 stand for age range from 70 to 80 years 6 age stand for age more than 80 years. Killip classification, $1=$ Killip I, 2= Killip II, 3= Killip III, 4=Killip IV. EF, 0 stands for $>50 \%, 1$ stands for less than $50 \%$. History of CABG, $1=$ with, $0=$ without; type of lesion, $1=$ Denovo lesion, $2=$ restenosis, $3=$ stent thrombosis; complete revascularization, $0=$ without, $1=$ with; mutivessel disease, $1=$ with, $0=$ without. Histogram refers to the score distribution in the derivation cohort. For the variables selected in the nomogram model, the values of different variables can correspond to different scores on the integral line at the top of the nomogram (the score range is $0-160$ points) through the projection of the vertical line, and the total score can be obtained by adding up the scores corresponding to the values of each variable. The cumulative occurrence probability of MACCE in 30 days, 3 year, 5 year and 7 years can be obtained from the total score on the prediction line at the bottom of the nomogram. Figure 2E, Elements of clinical prediction score and distribution of score among high-risk MI patients who undergent PPCI Figure 2D, The left side has shown the clinical prediction score variable and corresponding points. The right side has shown the graph of distribution of the clinical prediction score. 
Figure 3A

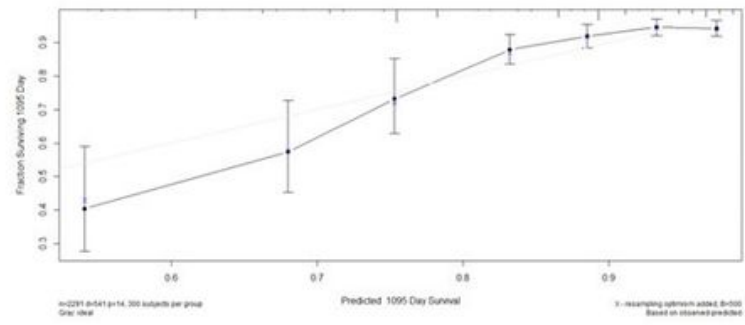

risk score calibration in the derivation cohort and the internal validation cohort; the stroke

events risk score of 3 -year

Figure 3B

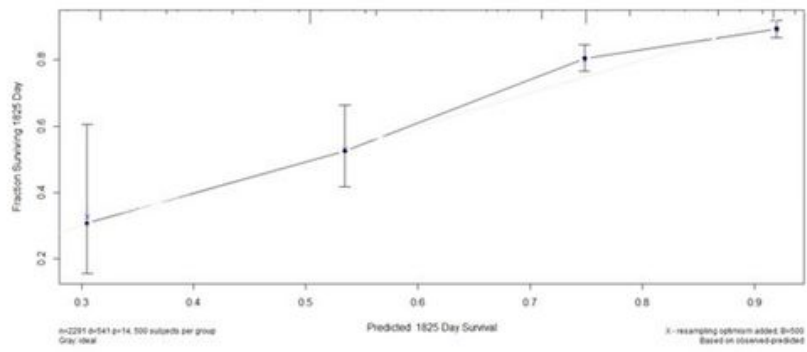

risk score calibration in the derivation cohort and the internal validation cohort; the stroke events risk score of 5-year

Figure $3 \mathrm{C}$

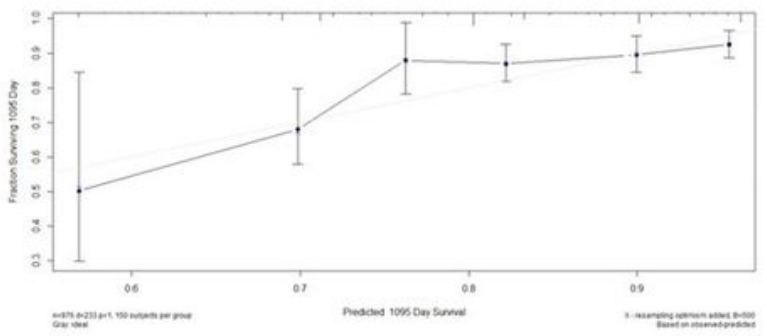

risk score calibration in the validation cohort and the internal validation cohort; the stroke events risk score of 3-year

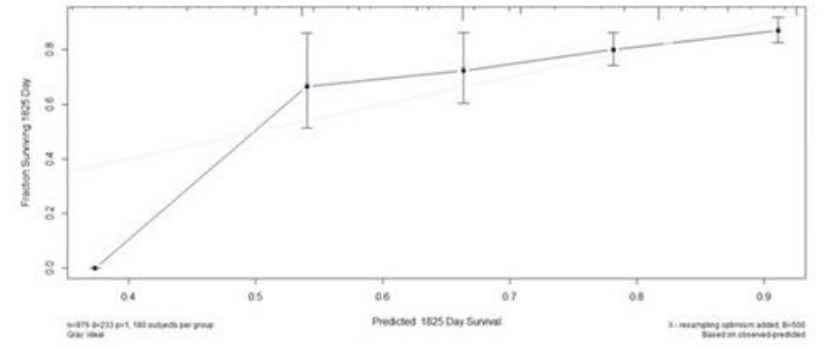

risk score calibration in the validation cohort and the internal validation cohort; the stroke events risk score of 5-year

Figure $3 E$ ROC curve of derivation cohort

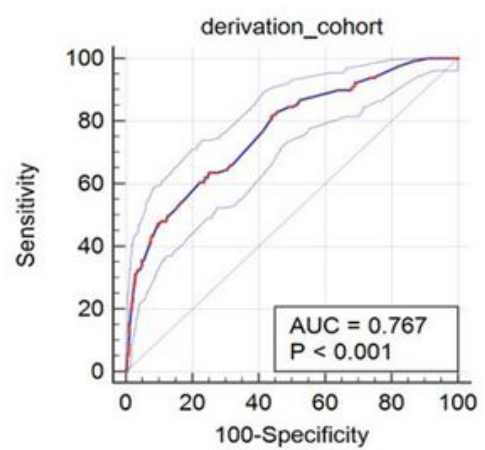

Figure $3 E$ showed the survival ROC curve of derivation cohort ( $A \cup C=0.767$, $p<0.001$ ). AUC, area under the curve; ROC, survival receiver operating characteristic; TP, true positive; FP, false positive.

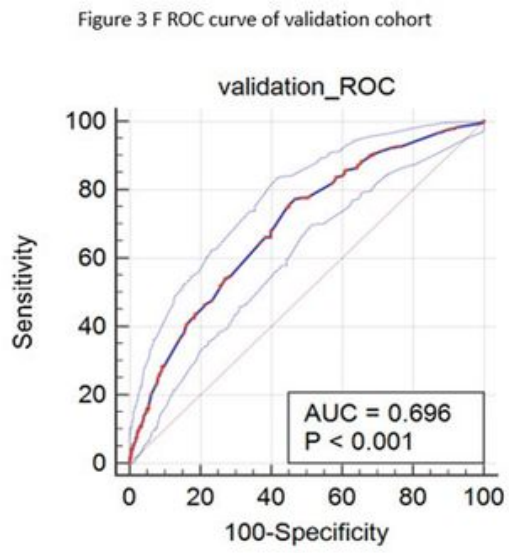

Figure $3 F$ showed the survival ROC curve of validation cohort ( $A \cup C=0.696, p<0.001$ ). AUC, area under the curve; ROC, survival receiver operating characteristic; TP, true positive; FP, false positive.

\section{Figure 3}

The internal validation of the model Figure $3 A-D$, risk score calibration in the derivation cohort and the internal validation cohort; the stroke events risk score of 3-year (A) 5-year (B) in the derivation cohort and 3-year (C) 5-year (D) in the validation cohort. Calibration is shown as the estimated risk against survival from Kaplan- Meier analysis. Gray line=perfect calibration. Figure 3E showed the survival ROC curve of derivation cohort $(A U C=0.767, p<0.001)$. Figure $3 F$ showed the survival ROC curve of validation cohort 
(AUC=0.696, $p<0.001$ ). AUC, area under the curve; ROC, survival receiver operating characteristic; TP, true positive; FP, false positive.
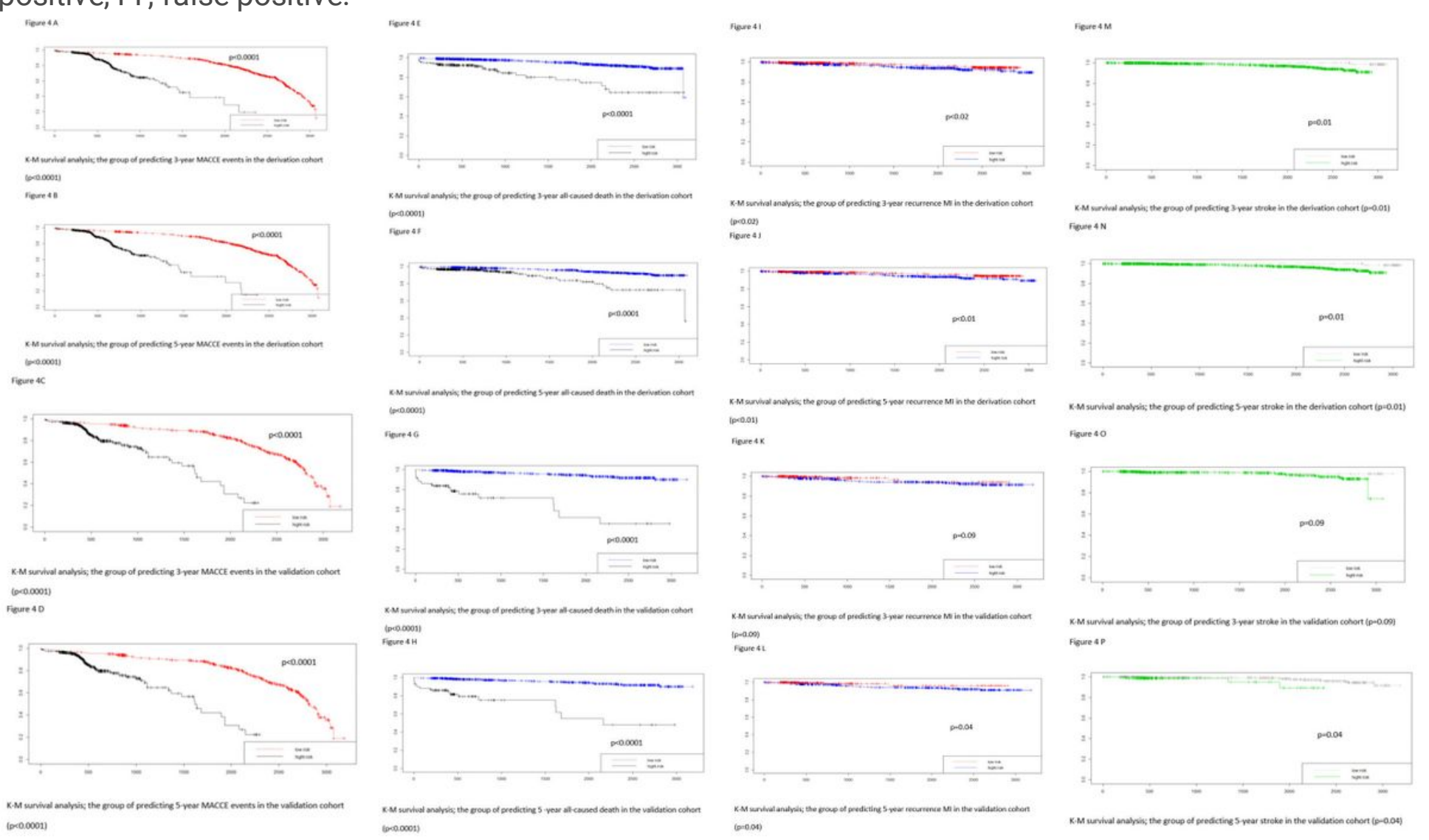

(6000)

\section{Figure 4}

K-M survival analysis; In the group of predicting MACCE events, the two groups displayed significant difference in both derivation cohort $(p<0.001)$ and validation cohort $(p<0.001)$ shown in figure 4A-D. In the subgroup of predicting all caused death, it is remarkable difference $(p<0.001)$ between the high risk group and relative low risk group in both development and validation group (figure $4 \mathrm{E}-\mathrm{H}$ ). Furthermore, when the endpoint was recurrence $\mathrm{MI}$, the longrank $\mathrm{p}$ was less than 0.02 in the 3-year derivation cohort and $\mathrm{p}$ less than 0.01 in the 5-year K-M curve in derivation cohort (figure 4I-L). Finally, we also found distinct discrepancy in predicting the stroke $(p<0.05)($ figure4 $M-P)$ events in 3-year and 5-year development and validation cohort.

\section{Supplementary Files}

This is a list of supplementary files associated with this preprint. Click to download.

- 20200702TripodChecklistPredictionModelDevelopmentMACCE.pdf

- Supplement.docx 